\title{
A note on embedding hypertrees
}

\author{
Po-Shen Loh* \\ Department of Mathematics \\ Princeton University \\ ploh@math.princeton.edu
}

Submitted: Jan 19, 2009; Accepted: May 19, 2009; Published: Jun 5, 2009

Mathematics Subject Classifications: 05C35, 05C65

\begin{abstract}
A classical result from graph theory is that every graph with chromatic number $\chi>t$ contains a subgraph with all degrees at least $t$, and therefore contains a copy of every $t$-edge tree. Bohman, Frieze, and Mubayi recently posed this problem for $r$-uniform hypergraphs. An $r$-tree is a connected $r$-uniform hypergraph with no pair of edges intersecting in more than one vertex, and no sequence of distinct vertices and edges $\left(v_{1}, e_{1}, \ldots, v_{k}, e_{k}\right)$ with all $e_{i} \ni\left\{v_{i}, v_{i+1}\right\}$, where we take $v_{k+1}$ to be $v_{1}$. Bohman, Frieze, and Mubayi proved that $\chi>2 r t$ is sufficient to embed every $r$-tree with $t$ edges, and asked whether the dependence on $r$ was necessary. In this note, we completely solve their problem, proving the tight result that $\chi>t$ is sufficient to embed any $r$-tree with $t$ edges.
\end{abstract}

\section{Introduction}

An $r$-graph is a hypergraph where all edges have size $r$, and a proper coloring is an assignment of a color to each vertex such that no edge is monochromatic. The chromatic number $\chi$ is the minimum $k$ for which there is a proper coloring with $k$ colors. A natural question is to investigate what properties can be forced by sufficiently large chromatic number. In the case of graphs, much is known, from trivialities such as $\chi>t$ implying the existence of a subgraph with all degrees at least $t$, to deeper results such as $\chi>4$ implying non-planarity. Far less is known for hypergraphs, but a folklore observation (see, e.g., [3]) is that whenever $\chi>2$, there is a pair of edges that intersect in a single vertex. This structure corresponds to a 2-edge hypertree, which in general is a connected hypergraph with no pair of edges intersecting in more than one vertex, and no sequence

${ }^{*}$ Research supported in part by a Fannie and John Hertz Foundation Fellowship, an NSF Graduate Research Fellowship, and a Princeton Centennial Fellowship. 
of distinct vertices and edges $\left(v_{1}, e_{1}, \ldots, v_{k}, e_{k}\right)$ with all $e_{i} \ni\left\{v_{i}, v_{i+1}\right\}$, where we take $v_{k+1}$ to be $v_{1}$.

For graphs, $\chi>t$ implies that there is a subgraph with all degrees at least $t$, in which we can embed any $t$-edge tree. Bohman, Frieze, and Mubayi recently posed the problem of generalizing this result to $r$-graphs. As they noted, this is not entirely trivial because there are hypergraphs with arbitrarily large minimum degree, but no copy of the path with 3 edges. Indeed, consider the 3 -graph with vertex set $\left\{v_{1}, \ldots, v_{n}\right\}$ and edges consisting of all triples containing $v_{1}$.

Observe that an $r$-uniform hypertree (henceforth referred to as an $r$-tree) with $t$ edges always has exactly $1+(r-1) t$ vertices. So, the complete $r$-graph on $(r-1) t$ vertices does not contain any $r$-tree with $t$ edges, while its chromatic number is exactly $t$. On the other hand, Bohman, Frieze, and Mubayi proved in [1] that every $r$-graph with $\chi>2 r t$ contains a copy of every $r$-tree with $t$ edges. They believed that their bound was far from the truth, and remarked at the end of their paper that it would be interesting to determine whether it should depend on $r$ in an essential way. In this note, we completely solve their problem, proving the following tight result.

Theorem 1 Every $r$-uniform hypergraph with chromatic number greater than $t$ contains a copy of every $r$-uniform hypertree with $t$ edges.

\section{Proof}

It suffices to show that for any $r$-tree $T$ with $t$ edges, every $T$-free $r$-graph $H$ can be properly colored with the integers $\{1, \ldots, t\}$. Although the proof is short, the following special case helps to illuminate the argument. Suppose the $r$-tree $T$ is a path with $t$ edges, and there is a proper $t$-coloring of $H-e_{1}^{*}$, the hypergraph on the same vertex set but with an arbitrary edge $e_{1}^{*}$ removed. The edge $e_{1}^{*}$ is monochromatic, say in color 1 , or else we are done. Let $v_{1}^{*}$ be an arbitrary vertex of $e_{1}^{*}$. Either we can recolor $v_{1}^{*}$ in color 2 without making any edge monochromatic in color 2 (and hence are done because $e_{1}^{*}$ is no longer monochromatic), or else some edge $e_{2}^{*} \ni v_{1}^{*}$ has all vertices except $v_{1}^{*}$ colored 2 . Note that since all vertices in $e_{2}^{*}$ are colored 2 except for $v_{1}^{*}$, and all vertices in $e_{1}^{*}$ are colored 1 , the two edges intersect only at $v_{1}^{*}$, thus forming a copy of the 2-edge path.

Suppose for a moment that $e_{2}^{*}$ is the unique edge containing $v_{1}^{*}$ which has all vertices except $v_{1}^{*}$ colored 2. Repeating the argument, we select $v_{2}^{*} \in e_{2}^{*}$, and either find an edge $e_{3}^{*} \ni v_{2}^{*}$ with all other vertices colored 3 (thus forming a 3-edge path together with $e_{2}^{*}$ and $e_{1}^{*}$ ), or obtain a proper coloring of $H$ by recoloring $v_{2}^{*}$ with color 3 and $v_{1}^{*}$ with color 2 . Unfortunately, when $e_{2}^{*}$ is not unique, the recoloring of $v_{1}^{*}$ with color 2 may make another edge monochromatic, so a more careful argument is needed in general. Nevertheless, for illustration only, let us make the simplifying uniqueness assumption, and continue in this way to find successively longer paths $e_{1}^{*}, e_{2}^{*}, \ldots, e_{s}^{*}$. Yet $H$ has no $t$-edge path, so this must stop before we need to use $t+1$ colors. Then, we will be able to properly $t$-color $H$ by recoloring each vertex $v_{i}^{*}$ with color $i+1$. 
Proof of Theorem 1. Let $T$ be an $r$-tree with $t$ edges. We will show that every $T$-free $r$-graph $H$ can be properly colored with the integers $\{1, \ldots, t\}$. Preprocess $T$ by labeling its edges and coloring its vertices as follows. Let $e_{1}$ be an arbitrary edge of $T$, and label the other edges with $e_{2}, \ldots, e_{t}$ such that for each $i \geq 2$, all edges $e_{j}$ along the (unique) path linking $e_{i}$ and $e_{1}$ are indexed with $j<i$. This can be done by exploring $T$ via breadth-first-search, for instance. Then, color each vertex $v \in T$ with the integer equal to the minimal index $i$ for which $e_{i} \ni v$.

We now induct on the number of edges of $H$. Let $e_{1}^{*}$ be an edge of $H$, and suppose that there is a proper $t$-coloring of $H-e_{1}^{*}$, the hypergraph on the same vertex set, but without the edge $e_{1}^{*}$. If this is already a proper coloring of $H$, then we are done. Otherwise, without loss of generality all vertices of $e_{1}^{*}$ received the color 1 . The following recoloring algorithm formalizes the above heuristic.

1. Let $H^{\prime} \subset H$ be a maximal colored-copy of a subtree of $T$ containing $e_{1}$, and let $T^{\prime} \subset T$ be that subtree. This means there is a color-preserving injective graph homomorphism $\phi: T^{\prime} \rightarrow H$ with maximal $T^{\prime} \ni e_{1}$, which exists because $e_{1}^{*}$ itself is a colored-copy of $e_{1}$.

2. Since $H$ is $T$-free, there is an edge $e_{s}$ in $T$ but not $T^{\prime}$, which is incident to some vertex $v \in T^{\prime}$. Change the color of $\phi(v) \in H$ to $s$. Terminate if $\phi(v) \in e_{1}^{*}$; otherwise, return to step 1.

The maximality of $H^{\prime}$ ensures that the recoloring step never creates any new monochromatic edges. Indeed, suppose for contradiction that $H$ has an edge $e^{\prime} \ni \phi(v)$ with all vertices except $\phi(v)$ colored $s$. Our preprocessing of $T$ ensures that no vertex in the colored-copy $H^{\prime}$ of $T^{\prime}$ has color $s$, so $e^{\prime}$ intersects $H^{\prime}$ only at $\phi(v)$. Thus $H^{\prime}+e^{\prime}$ would be a colored copy of $T^{\prime}+e_{s}$, contradicting maximality.

Also, the algorithm terminates because the recoloring step always increases the (integer) color of $\phi(v)$, but no color ever exceeds $t$. To see this, observe that since we had a colored-copy, the color of $\phi(v)$ originally equalled the color of $v \in T$, which we defined to be the minimal index $i$ such that $e_{i} \ni v$. By our preprocessing of $T, e_{s} \notin T^{\prime}$ implies that some lower-indexed edge also contains $v$. Hence $\phi(v)$ indeed had color less than $s$. Therefore, we eventually obtain a proper coloring of $H$.

\section{Concluding remarks}

- The standard proof of the graph case of Theorem 1 uses the fact that every $t$-edge tree can be embedded in any graph with minimum degree at least $t$. This is not true for hypergraphs, so our proof uses a completely different argument that does not rely on degrees at all. Consequently, our proof also gives a new perspective on the graph case.

- Results for graphs that used $\chi>t$ to embed $t$-edge trees can now be extended to uniform hypergraphs. Consider, for example, the following classical result of 
Chvátal, referred to as "one of the most elegant results of Graph Ramsey Theory" by Graham, Rothschild, and Spencer in their book [4]. The Graph Ramsey number $R\left(H_{1}, H_{2}\right)$ is the smallest $n$ such that every red-blue edge-coloring of $K_{n}$ contains either a red copy of $H_{1}$ or a blue copy of $H_{2}$. When $H_{1}$ is a complete graph $K_{k}$ and $H_{2}$ is any $t$-edge tree, Chvátal determined that $R\left(H_{1}, H_{2}\right)$ is precisely $(k-1) t+1$.

Using Theorem 1, we can lift one of the standard proofs of this result to $r$-graphs. Indeed, suppose we have a red-blue edge-coloring of the complete $r$-graph on $(k-$ 1) $t+1$ vertices, and let $H$ be the hypergraph on the same vertex set formed by taking only the blue edges. If $\chi(H) \leq t$, then $H$ has an independent set of size at least $\left\lceil\frac{(k-1) t+1}{t}\right\rceil=k$, which corresponds to a red complete $r$-graph on that many vertices. Otherwise, if $\chi(H)>t$, then Theorem 1 implies that any $t$-edge tree can be found in the blue graph $H$.

On the other hand, the $r$-graph obtained by taking the disjoint union of $\left\lfloor\frac{k-1}{r-1}\right\rfloor$ copies of the complete $r$-graph on $(r-1) t$ vertices does not contain any $r$-tree with $t$ edges, while its independence number is at most $k-1$. So, if we color all of its edges blue, and add in all missing edges with color red, then we obtain an edge-coloring of the complete $r$-graph on $\left\lfloor\frac{k-1}{r-1}\right\rfloor \cdot(r-1) t$ vertices with no red $K_{k}^{(r)}$ and no blue $r$-tree with $t$ edges. Therefore, the $r$-graph result is tight when $r-1$ divides $k-1$, and asymptotically tight for $k \gg r$.

Acknowledgment. The author thanks his Ph.D. advisor, Benny Sudakov, for introducing him to this problem, and for remarks that helped to improve the exposition of this note. Also, he thanks Asaf Shapira for pointing out the application of the main theorem to Chvátal's result, and the referee for carefully reading this article.

\section{References}

[1] T. Bohman, A. Frieze, and D. Mubayi, Coloring $H$-free hypergraphs, Random Structures and Algorithms, to appear.

[2] V. Chvátal, Tree-complete Ramsey numbers, Journal of Graph Theory 1 (1977), 93.

[3] P. Erdős and L. Lovász, Problems and results on 3-chromatic hypergraphs and some related questions, Infinite and finite sets (Colloq., Keszthely, 1973; dedicated to P. Erdös on his 60th birthday), Vol. II, pp. 609-627. Colloq. Math. Soc. János Bolyai, Vol. 10, North-Holland, Amsterdam, 1975.

[4] R. Graham, B. Rothschild, and J. Spencer, Ramsey Theory, 2nd ed., Wiley, New York (1980). 